\title{
Supracostal Access: Does it affect Tubeless Percutaneous Nephrolithotomy Efficacy and Safety?
}

\author{
J. Jun-Ou, Bannakij Lojanapiwat
}

Division of Urology, Department of Surgery, Chiangmai University, Chiangmai, Thailand

\begin{abstract}
Introduction: Tubeless percutaneous nephrolithotomy (PCNL) in selected patients has been found to be safe and can reduce postoperative discomfort without increasing complications. The challenges of tubeless PCNL via supracostal access are inadequate drainage and postoperative bleeding, conditions that may increase pulmonary complications. We compare the efficacy and safety of the tubeless supracostal versus the standard supracostal PCNL.

Materials and Methods: Supracostal PCNL with one percutaneous renal access, no significant bleeding, extravasation and residual stone was performed in 95 patients. Of these, 43 were tubeless PCNL (Group-I) and 52 were PCNL with standard routine postoperative nephrostomy tube (Group-II). In group-I, PCNL was done by the standard supracostal technique with the placement of a postoperative external ureteral catheter for 48 hours. The operative time, success rate, hospital stay and ensuing complications were compared between group-I and group-II.

Results: Patients in the tubeless PCNL group (Group-I) were $90.7 \%$ stone -free while those with standard routine postoperative nephrostomy tube(Group-II) were $84.6 \%$ stone -free. Additionally, stone fragments of less than $4 \mathrm{~mm}$ in diameter were found in $9.3 \%$ of patients in group-I and $25.4 \%$ in group-II. The success rate, hematocrit change and complication were not significantly different between both groups. The analgesic requirement, operative time and hospital stay were all significantly less in the tubeless supracostal group (Group-I). None of group I and only one patient of group II needed intercostal drainage.

Conclusion: Tubeless supracostal percutaneous nephrolithotomy in selected patients is effective with acceptable complications. This technique offers the advantage of lower analgesic requirement, shorter operative time and hospital stay. The pulmonary complication is the same as the standard supracostal percutaneous nephrolithotomy.
\end{abstract}

Key words: percutaneous nephrolithotomy; efficacy; complications

Int Braz J Urol. 2010; 36: 171-6

\section{INTRODUCTION}

Percutaneous nephrolithotomy (PCNL) is the accepted treatment for large renal and upper ureteral stones. The four stages of PCNL are: (1) renal access, (2) tract dilatation, (3) nephroscopy and stone disintegration, and (4) nephrostomy tube placement. In uncomplicated PCNL where there is no significant extravasation, significant bleeding, or any need for a second nephroscopy, the placement of the nephrostomy tube may not be necessary (tubeless PCNL) (1-4). In specific situations of PCNL, a supracostal renal approach is necessary (5-8). Pulmonary complication is more common with this approach due to the anatomy of the kidney. Extravasation and bleeding may be more commonly found in tubeless PCNL and 
may increase the incidence of postoperative pulmonary complications in supracostal access. The efficacy and complication of tubeless PCNL via the supracostal route were compared to those of supracostal PCNL with routine nephrostomy tube placement.

\section{MATERIALS AND METHODS}

\section{Patients}

A total of 95 patients underwent PCNL via supracostal. The patients were divided into two groups, 43 receiving tubeless supracostal PCNL (Group-I) and 52 receiving supracostal PCNL with routine nephrostomy tube placement (Group-II). Four criteria were established for PCNL of both groups in this study regardless of the stone burden, namely, (1) a single access site, (2) non obstructive renal unit, (3) no significant perforation or bleeding, and (4) a second look would not be required. The mean age of group-I and II was $51.49 \pm 12.77$ years (range $30-83$ years) and $50.63 \pm 12.18$ years (range 23-76 years), respectively. The mean stone size of group I and II was $3.83 \pm 1.45 \mathrm{~cm}$ (range 1.8-8.0 cm) and $4.11 \pm$ $1.57 \mathrm{~cm}$ (range $2.3-9.5 \mathrm{~cm}$ ), respectively. Ten patients $(23.8 \%)$ in group I and 12 patients (23.1\%) in group II had previous open nephrolithotomy. Patient profiles are shown in Table-1. The positions of the calculi are shown in Table-2. All patients were operated by a single surgeon.

\section{Methods}

Single stage percutaneous nephrolithotomy was done in all patients. Intravenous antibiotic was given before the operation in all cases. After the induction of general anesthesia, an open-end $6 \mathrm{~F}$ ureteral catheter was placed via a transurethral approach into the ureter with the patient in a supine position. The tip of the ureteral catheter was placed at the ureteropelvic junction or at the renal pelvis. The percutaneous access was created by a single urologist (BL) in all cases. Under fluoroscopic guidance in the prone position and after injection of contrast media via ureteral catheter, 95 sites were supracostal upper pole access. The needle was pushed through the diaphragm and retroperitoneum in full expiration, whereas the needle was passed through the kidney during deep inspiration. The working and safety guide-wires were inserted after the tip of the needle was in the collecting system. Tract dilatations were performed by Amplatz fascial dilators (Cook Urological Spencer, Indiana, USA) or telescopic metal dilators sizes from 8F-30F, with an inserted 30F Amplatz sheath. Using a standard nephroscope (26F), stone disintegration was obtained with ultrasonic and/or pneumatic lithotripsy. Fluoroscopy and contrast nephrostogram with systematic nephroscopy were performed to evaluate the stone-free status.

As regards tubeless PCNL, the ureteral catheter (the same $6 \mathrm{~F}$ ureteral catheter that was placed at the beginning of the operation) was adjusted neph-

Table 1 - Profiles of patients.

\begin{tabular}{lccc}
\hline & $\begin{array}{c}\text { Group I } \\
\text { (Supracostal Tubeless PCNL) }\end{array}$ & $\begin{array}{c}\text { Group II } \\
\text { (Supracostal Standard PCNL) }\end{array}$ & p Value \\
\hline Patients & 43 & 52 & \\
Side (R:L) & $19: 24$ & $23: 29$ & \\
Gender (M:F) & $20: 23$ & $38: 14$ & \\
Age (years) & $51.49 \pm 12.77$ & $50.63 \pm 12.18$ & 0.740 \\
& $($ range $30-83)$ & (range 23-76) & \multirow{2}{*}{0.363} \\
Stone size (cm) & $3.83 \pm 1.45$ & $4.11 \pm 1.57$ & \multirow{2}{*}{ (range 2.3-9.5) } \\
Previous open nephrolithotomy & $($ range $1.8-8.0)$ & $12(23.1 \%)$ & 0.934 \\
\hline
\end{tabular}


Table 2 - Stone positions.

\begin{tabular}{lccc}
\hline & $\begin{array}{c}\text { Group I } \\
\text { (Supracostal tubeless PCNL) }\end{array}$ & $\begin{array}{c}\text { Group II } \\
\text { (Standard supracostal PCNL) }\end{array}$ & p Value \\
\hline Staghorn stone (\%) & $14(32.5)$ & $15(28.8)$ & 0.227 \\
Calyceal stone (\%) & $12(27.9)$ & $9(17.3)$ & \\
Pelvic + Calyceal stone (\%) & $13(30.2)$ & $25(48.1)$ & \\
Upper ureteral stone (\%) & $2(4.7)$ & $3(5.8)$ & \\
Upper ureteral stone + calyceal stone (\%) & $2(4.7)$ & 0 & \\
\hline
\end{tabular}

roscopically, the tip being placed at the renal pelvis. The working sheath was removed with the safety guide wire still in placed. The nephrostomy site was examined and, if there was no evidence of active bleeding for 5 minutes, the wound was closed with sutures. The guide wire was then removed and the ureteral catheter was left attached to the Foley catheter for 48 hours. The nephrostomy tube sized 20F was routinely inserted in the remained cases (Group-II). The prolong placement of the ureteral catheter and nephrostomy tube depended on postoperative fever, bleeding or other complications.

Before and on the first day of the surgery, all patients were tested for complete blood count to determine any change in the hematocrit level. Postoperative chest $\mathrm{X}$-ray (CXR) was routinely done in all cases. The patient's symptoms and CXRs were used to evaluate pulmonary complications. If the patients developed shortness of breath, chest pain and desaturation in the recovery room and CXR revealed pleural effusion, the intercostal drainages were done immediately. Postoperative plain film KUB at day 1 was done for evaluation of the stone free status. Meperidine injection was given when the patients complained of pain. Statistical analysis with Chi-square for qualitative variables and Student's-t-tests for quantitative variables with $\mathrm{p}<0.05$ was considered statistically significant.

\section{RESULTS}

The stone-free rate was $90.7 \%$ in group I and $84.6 \%$ in group II, and fragments $\leq 4 \mathrm{~mm}$ occurred $9.3 \%$ and $15.4 \%$ in groups I and II, respectively. The operative time was $47.38 \pm 16.93 \mathrm{~min}$ (range $25-90$ min) in group I and 58.85 \pm 18.46 (range 30-105 $\mathrm{min}$ ) in group II, which was significantly different $(\mathrm{p}=0.03)$. The hospital stay was $3.45 \pm 1.01$ (range 2-7 days) and $4.83 \pm 1.44$ (range 3-10 days) in group I and group II, respectively. Meperidine usage was $37 \pm 31(0-150) \mathrm{mg}$ in group I and $70 \pm 36(0-150)$ in group II which was significantly different $(\mathrm{p}<$ 0.001 ). Decrease in the hematocrit level was $2.65 \pm$ $2.59 \mathrm{mg} \%$ in group I and $2.31 \pm 2.46 \mathrm{mg} \%$ which was not statistically different $(\mathrm{p}=0.522)$. There were 4 patients $(9.30 \%)$ in group I and 5 patients $(9.62$ $\%$ ) in group II who had hydrothorax as indicated by respiratory symptoms or postoperative CXRs Only 1 patient $(1.92 \%)$ of group II needed intercostal drainage (Table-3).

\section{COMMENTS}

Percutaneous nephrolithotomy has replaced open stone surgery for large renal or upper ureteral calculi because it is a less minimally invasive technique. The last stage after completion of PCNL is the placement of the nephrostomy tube. The purpose of the nephrostomy tube is to provide hemostasis along the tract, avoid urinary extravasation and maintain adequate drainage of the kidney. In selected patients, tubeless percutaneous nephrolithotomy, with only an externalized ureteral catheter or double J stent, is safe, economical and provides reduced postoperative discomfort with the same outcome (1-4). Inclusion criteria of tubeless PCNL are the use of a single access site where the renal unit is not obstructive, no significant perforation, bleeding and no need for a 
Table 3 - Results and complications.

\begin{tabular}{lccc}
\hline & $\begin{array}{c}\text { Group I } \\
\text { (Supracostal tubeless PCNL) }\end{array}$ & $\begin{array}{c}\text { Group II } \\
\text { (Standard supracostal PCNL) }\end{array}$ & p Value \\
\hline Stone free (\%) & $39(90.7)$ & $44(84.6)$ & 0.131 \\
Stone fragment $\leq 4 \mathrm{~mm}(\%)$ & $4(9.30)$ & $7(15.4)$ & \\
Operative time (min) & $47.38 \pm 16.93$ & $58.85 \pm 18.46$ & 0.030 \\
& $($ range 25-90) & $($ range 30-105) & $<0.001$ \\
Hospital stay (days) & $3.45 \pm 1.01$ & $4.83 \pm 1.44$ & $<0.001$ \\
& $($ range 2-7) & $70.00 \pm 36.00$ & 0.522 \\
Meperidine usage (mg) & $37.00 \pm 31.00$ & $($ range 0-150) & 0.492 \\
Hematocrit change (mg\%) & $($ range 0-150) & $2.3 \pm 2.46$ & $5(9.62)$ \\
Pleural complication (\%) & $2.65 \pm 2.59$ & $1(1.92)$ & \\
Intercostal drainage & $4(9.30)$ & - &
\end{tabular}

second look (1-4). The stone burden may not necessarily to be taken into account.

Winfield and associates reported significant complications after premature removal of nephrostomy tube after PCNL (9). Bellman and colleagues reported tubeless PCNL with only a double-J stent for one week without compromising efficacy and safety (1). We previously reported tubeless PCNL in 37 patients with only the placement of an externalized ureteral catheter for 48 hours to provide adequate drainage for the upper tract without increasing complications and blood transfusion (2). Due to the selected patients in tubeless PCNL, the stone free status had to be assessed during the operation. The systematic nephroscopy, intraoperative fluoroscopy with contrast nephrostogram were used for evaluation of the stone free status. Karami and Gholamrezaie (10) and Aghamir et al. (11) reported the technique of tubeless PCNL without any externalized ureteral catheter or double $\mathrm{J}$ stent. They found that the totally tubeless PCNL technique was safe and effective, requires less hospital stay and analgesics and led to a fast recovery time. No urinoma was found on postoperative ultrasound with an average length of hospital stay of 1.6 days.

Under specific conditions, access to the kidney may require the upper pole approach. The indications for the upper pole approach are staghorn calculi, large or multiple upper calyceal stones, renal calculi associated with ureteropelvic junction or upper ureteral pathology, large upper ureteral calculi and calculi in specific anatomy $(8,12,13)$. The upper pole approach provides a straight tract along the long axis of the kidney and ensures reaching most of the collecting system, which provides easier manipulation of the rigid nephroscope and other rigid instruments. This approach can achieve a better stone clearance $(12,13)$. Upper pole access can be achieved via the supracostal and infracostal approaches. Due to the anatomy of the kidney, pulmonary complications are more common with the supracostal approach $(8,13)$. We previously reported 170 supracostal PCNL compared with 294 infracostal PCNL. We found that both approaches provide the same effective results, but pulmonary complications are higher when using the supracostal approach as compared with the subcostal approach. The pulmonary complications that needed intercostal drainage were $5 \%$ and $0.3 \%$ in supracostal and subcostal approach, respectively. There was a 17 -fold greater possibility of pulmonary complication in the supracostal when compared to the subcostal approach (12).

Postoperative pulmonary complications after PCNL can be detected by postoperative symptoms and 
postoperative CXRs. The symptoms of pulmonary complications are poor oxygen saturation, dyspnea and tachypnea postoperatively. The abnormality of postoperative CXRs depends on the volume of pleural effusion (14). The treatment of hydrothorax depends on the amount of hydrothorax and the patients symptoms. Conservative treatment is preferentially for those with no or mild symptoms and minimal effusion. Patients with significant symptoms and a large amount of pleural effusion need intercostal drainage. The incidence of pulmonary complications after 12th supracostal approach that need surgical intervention was from $0 \%$ to $23 \%$ (range $5-8$ ).

The techniques of supracostal approach require coordination with the anesthetist to control respiration. To avoid injuring the intercostal vessels, the intercostal puncture is made in the lower half of the intercostal space. During full expiration, the needle is passed through the retroperitoneum and diaphragm to prevent injury to the lung. The needle insertion, which is passed through the parenchyma to the collecting system, is done during deep inspiration for downward displacement of the kidney. An Amplatz sheath is used in all patients during the percutaneous supracostal approach to maintain low pressure irrigation that can reduce the risk of pleural effusion and extravasation. After supracostal access, postoperative CXRs were routinely used in all cases for evaluation of pulmonary complications (12).

One concern with the tubeless PCNL technique is to ensure adequate drainage and no significant bleeding postoperatively. Extravasation and bleeding can be significant problems of this technique after supracostal approach and may lead to pulmonary complications. There is limited published data in the literature to date on tubeless PCNL in percutaneous nephrolithotomy via supracostal access. Shah and colleagues (15) reported 72 patients of tubeless PCNL via supracostal access compared with 72 patients with routine standard supracostal PCNL. Only a single $6 \mathrm{~F}$ double $\mathrm{J}$ stent was placed in the tubeless group, whereas both the $6 \mathrm{~F}$ double $\mathrm{J}$ stent and a $28 \mathrm{~F}$ nephrostomy tube were placed in control group. The double J stent was removed at 1-2 weeks after the surgery and nephrostomy tube was removed in 12-24 hours. Stone free at 1 to 2 weeks was $99.44 \%$ and $91.66 \%$ in tubeless and in control group, respec- tively. Blood transfusion was required in 3 patients of the tubeless group and in 4 of the control group with only 1 patient in control group had hydrothorax that needed intervention. They concluded that tubeless PCNL via supracostal is safe and effective with lower analgesic requirement and shorter hospital stay without increasing thoracic complication. Shah and associates (16) reported 30 bilateral simultaneous supracostal tubeless accesses in 51 urinary tracts of 45 renal units. As regards the supracostal access tracts, no urine leakage or major chest complication were found, and patients were stone free or had residual stone fragment of less than $5 \mathrm{~mm}$ in 39 and 4 renal units, respectively. Gonen et al. reported 10 tubeless and stentless supracostal PCNL. These authors found that this technique is safe and offers advantages of a lower analgesia and shorter hospital stay without increasing of pulmonary complication (17).

In our series, all patients in the present study had the same criteria for the tubeless PCNL. We found that the incidence of pulmonary complications in tubeless supracostal PCNL was not different from the standard supracostal PCNL. The pleural complication was $9.30 \%$ in tubeless supracostal PCNL and $9.62 \%$ in standard PCNL. Almost all of these patients were resolved after conservative treatment. Only one standard PCNL patient needed intercostal drainage. The results of the treatment, as justified by stone free and insignificant residual fragment condition, were the same in both groups. The analgesic requirement, operating time and hospital stay were less in tubeless group compared to the standard supracostal PCNL. All patients in both groups, who had previous open nephrolithotomy (10 of group I and 12 of group II) were found to be safe with the same outcome. The longer hospital stay in our series compared to the other studies was obviously related to our tubeless PCNL technique which was different from the other patients where we routinely left a ureteral catheter indwelling for 48 hours after the surgery (2).

\section{CONCLUSIONS}

Tubeless supracostal percutaneous nephrolithotomy in selected patients with externalized ureteral catheter is safe and effective with lower analgesic re- 
quirement, shorter operative time and shorter hospital stay. This technique does not increase hemorrhage or pulmonary complications when compared with the standard supracostal percutaneous nephrolithotomy.

\section{CONFLICT OF INTEREST}

None declared.

\section{REFERENCES}

1. Bellman GC, Davidoff R, Candela J, Gerspach J, Kurtz S, Stout L: Tubeless percutaneous renal surgery. J Urol. 1997; 157: 1578-82.

2. Lojanapiwat B, Soonthornphan S, Wudhikarn S: Tubeless percutaneous nephrolithotomy in selected patients. J Endourol. 2001; 15: 711-3.

3. Goh M, Wolf JS Jr: Almost totally tubeless percutaneous nephrolithotomy: further evolution of the technique. J Endourol. 1999; 13: 177-80.

4. Shah HN, Kausik VB, Hegde SS, Shah JN, Bansal MB: Tubeless percutaneous nephrolithotomy: a prospective feasibility study and review of previous reports. BJU Int. 2005; 96: 879-83.

5. Stening SG, Bourne S: Supracostal percutaneous nephrolithotomy for upper pole caliceal calculi. J Endourol. 1998; 12: 359-62.

6. Golijanin D, Katz R, Verstandig A, Sasson T, Landau EH, Meretyk S: The supracostal percutaneous nephrostomy for treatment of staghorn and complex kidney stones. J Endourol. 1998; 12: 403-5.

7. Munver R, Delvecchio FC, Newman GE, Preminger GM: Critical analysis of supracostal access for percutaneous renal surgery. J Urol. 2001; 166: 1242-6.
8. Gupta R, Kumar A, Kapoor R, Srivastava A, Mandhani A: Prospective evaluation of safety and efficacy of the supracostal approach for percutaneous nephrolithotomy. BJU Int. 2002; 90: 809-13.

9. Winfield HN, Weyman P, Clayman RV: Percutaneous nephrostolithotomy: complications of premature nephrostomy tube removal. J Urol. 1986; 136: 77-9.

10. Karami H, Gholamrezaie HR: Totally tubeless percutaneous nephrolithotomy in selected patients. J Endourol. 2004; 18: 475-6.

11. Aghamir SM, Hosseini SR, Gooran S: Totally tubeless percutaneous nephrolithotomy. J Endourol. 2004; 18: 647-8.

12. Lojanapiwat B, Prasopsuk S: Upper-pole access for percutaneous nephrolithotomy: comparison of supracostal and infracostal approaches. J Endourol. 2006; 20: 491-4.

13. Lang EK, Thomas R, Davis R, Colon I, Cheung W, Sethi E, et al.: Risks and benefits of the intercostal approach for percutaneous nephrolithotripsy. Int Braz J Urol. 2009; 35: 271-81; discussion 281-3.

14. Woodring JH: Detection of pleural effusion on supine chest radiographs. AJR Am J Roentgenol. 1987; 149: 858-9.

15. Shah HN, Hegde SS, Shah JN, Bansal MB: Safety and efficacy of supracostal access in tubeless percutaneous nephrolithotomy. J Endourol. 2006; 20: 1016-21.

16. Shah HN, Kausik VB, Hegde SS, Shah JN, Bansal MB: Safety and efficacy of bilateral simultaneous tubeless percutaneous nephrolithotomy. Urology. 2005; 66: 500-4.

17. Gonen M, Cicek T, Ozkardes H: Tubeless and stentless percutaneous nephrolithotomy in patients requiring supracostal access. Urol Int. 2009; 82: 440-3.

\author{
Correspondence address: \\ Dr. Bannakij Lojanapiwat \\ Division of Urology \\ Department of Surgery \\ Chiangmai University \\ Chiangmai, 50200, Thailand \\ Fax: + 6653 945-154 \\ E-mail: blojanap@mail.med.cmu.ac.th
}

